\title{
Recommending Garment Products in E-Shopping Environment by Exploiting an Evolutionary Knowledge Base
}

\author{
Junjie Zhang ${ }^{1}$, Xianyi Zeng ${ }^{1,2,3}$, Ludovic Koehl ${ }^{2,3}$, Min Dong ${ }^{1}$ \\ ${ }^{1}$ Wuhan Textile University, 430073 Wuhan, China \\ ${ }^{2}$ Univ Lille Nord de France, F-59000 Lille, France \\ ${ }^{3}$ Laboratoire Génie et Matériaux Textile (GEMTEX), France
}

Received 7 August 2017

Accepted 20 October 2017

\begin{abstract}
Garment purchasing through the e-shopping platforms has become an important trend for consumers of all parts of the world. More and more e-shopping platforms have proposed recommendation functions to consumers in order to make them to obtain more easily desired products and then increase shopping sales. However, there are two main drawbacks in the existing recommendation systems. First, it systematically lacks feedback processing in these systems. If a consumer is not satisfied with the recommendation result, there is no self-adjustment function. The other drawback is that the existing recommendation systems are mostly closed, without considering the possibility of data and knowledge updating. Considering the above drawbacks, we propose a new recommendation system integrating the following features: 1) automatic adjustment of the knowledge according to the consumers' feedback, 2) making the system open and adaptive so that the consumer can easily add or replace criteria and data. This proposed recommendation system can effectively help consumers to choose garments on the Internet. Compared with the other systems, the proposed one is more robust and more interpretable owing to its capacity of handling uncertainty.
\end{abstract}

Key words: recommendation system, knowledge base, self-learning, human-machine interaction, feedback.

\section{Introduction}

With rapid development of e-commerce, more and more consumers buy garments via the e-shopping platforms. ${ }^{1}$ E-shopping is becoming a generally purchasing way due to its economical and convenient features. Recommendation systems have been widely developed in the situation of e-shopping platforms. ${ }^{2-3}$ In general, they can typically produce a ranking list of candidates using one of the two widely used methods, i.e. collaborative or content-based filtering. In general, three categories of recommendation systems have been developed. ${ }^{4}$

Collaborative filtering: Collaborative filtering methods are based on collecting and analyzing a large amount of information on users' behaviors, activities or preferences and predicting what users will like based on their similarity to other users. ${ }^{5}$

Content-based filtering: Content-based filtering methods are based on a description of the item and a profile of the user's preference. ${ }^{6}$

Hybrid Recommendation systems: Recent research has demonstrated that a hybrid approach, combining collaborative filtering and content-based filtering could be more effective in some cases. ${ }^{7-8}$

A large number of the shopping web sites already integrate recommender systems for helping their consumers to find relevant products in order to increase their sales. ${ }^{9}$ In the developed personalized recommender systems, data mining techniques, such as association 
rules mining for offline operations and connection rule excavation for online operations, have been widely used. ${ }^{10}$ In practice, in order to cope with data explosion on Internet and retrieve relevant and concise product information out of overflowing advertisement, the methodology of recommending and learning has been used for acquiring the user preference and providing user with the user adaptive product information. For fashion products, a large scale visual recommendation system has been proposed based on four data driven models: Complementary Nearest Neighbor Consensus, Gaussian Mixture Models, Texture Agnostic Retrieval and Markov Chain LDA. ${ }^{11}$ Markov Chain utilizes the initial probability distribution and state transition probability matrix in order to compute possible future states. It has been successfully used to forecast the garment fashion forecasting. ${ }^{12}$

Classical recommendation systems for e-shopping are mainly based on the consumer's past purchases, product ratings and demographic data without considering the profitability of the items being recommended. In this context, Das et al consider the question of how a vendor can directly incorporate the profitability of items into its recommendation system so as to maximize expected profit while still providing accurate recommendations. ${ }^{13}$ Sutar and Khade propose a system which will be able to recommend the user to choose appropriate outfits suits to their personality. The guidelines for selection of their respective outfits are based upon various human body parameters that evolve with integration or learning of available labeled and unlabeled data. The proposed system will have image capturing by using HAAR feature or input device for getting body parameters. They intend to classify and extract the best possible outfits from the system by using HIGEN MINER algorithm. ${ }^{14}$

Consumer's emotion is an important factor in classical shopping, especially for fashion products. However, few researchers have integrated emotions into recommender systems. Liu et al. propose a novel framework model for emotion based e-commerce recommendation systems. The core of the recommendation framework is the construction of the product and consumer emotion model by two-dimensional overlap spaces, which plays an important role in conveying emotions in products. The user's emotion in a tourist recommender system has been estimated using Emotion Generating Calculations and Mental State Transition Network. ${ }^{15}$ Also, a web chat room with emotions has been designed for perceiving e-learners' emotional states by means of interactive texts. And it recommends resources such as music and mottos to an e-learner when it detects negative emotional states. Meanwhile, it recommends emotion regulation cases to the e-learner's listeners and teachers. ${ }^{16}$

In summary, the current recommendation systems are generally based on experimental data only without systematically exploiting professional knowledge and consumer's emotions in shopping scenarios. Also, it systematically lacks feedback processing in these systems. If the consumer is not satisfied with the recommendation result, there is no self-adjustment function. Moreover, the existing recommendation systems are mostly closed, without considering the possibility of data and knowledge updating.

Considering the above drawbacks, we propose a new recommendation system possessing the following advantages:

i. It is a knowledge-based recommendation system. The rules of the knowledge base are extracted from four sensory experiments.

ii. It can adjust the knowledge base automatically according to the consumers' feedback. The classical single recommendation process has been transformed into a cycle of recommendation evaluation - adjustment.

iii. It is an open system so that the consumer can easily add or replace criteria and data. The knowledge base will evolve with new successful recommendation results.

The originalities of the contribution are described below. The propose system is based on a knowledge base characterizing the relations between consumer profiles (body shapes and fashion requirements expressed by style keywords and visual images) and product profiles of garments (fitting, details). Consumer perception and emotions on fashion products can be fully taken into account in these profiles. For correctly characterizing these relations, we propose a list of 8 normalized sensory criteria describing both consumer and product profiles. Moreover, fuzzy techniques have been used for formalizing and computing these relations according to the evaluations of consumers and shopping professionals. Also, these relations can be updated by 
integrating consumer's new evaluation data on recommended products.

This paper is organized as follows. In Section 2, we describe the details for implementation of the Knowledge-based Recommendation Module. Section 3 presents the details for implementation of the Knowledge Updating Module. In Section 4, the proposed system has been analyzed and validated by using an illustrative example on jeans e-shopping of female style. A conclusion is given in Section 5.

\section{The Knowledge-based Recommendation Module}

In this paper, we illustrate the system with a concrete case: online recommendation of jeans for Chinese women whose ages are from 18 to 25 years old. In this system, the specific consumer needs to input her profile, composed of: body data, style keywords and visual images.

For recommending relevant garments, there are two modules in this system, namely 1) the Knowledge-based Recommendation Module for providing more personalized products; 2) the Knowledge Updating Module, enabling to progressively improve the quality of the knowledge base according to the unsatisfied evaluation descriptors on currently recommended products.

\subsection{Formalization of the concepts and data}

We formalize the input and output variables of the proposed system as well as concerned data:

Category 1: Let $E=\{e 1, e 2, \ldots, e p\}$ be a set of $p(p=8)$ normalized evaluation criteria describing both products and consumers.

Category 2: Let $\mathrm{BS}=\{\mathrm{bs} 1, \ldots, \mathrm{bsm}\}$ be a set of $\mathrm{m}$ $(\mathrm{m}=20)$ body shapes, representing all the combinations of the 5 standard tall-low types and 4 fat-thin types obtained from the Chinese National Standard GB/T 1335.2-1997. We can describe the tall-low into five levels: X1: short, X2: a little short, X3: middle, X4: a little tall, X5: tall. We can define fat-thin as four levels: Y1: underweight Y2: normal, Y3: overweight, Y4: obese.

Category 3: Let $S=\{s 1, \ldots, s n\}$ be a set of $n$ style keywords ( $\mathrm{n}=8)$, including "Elegant", "Feminine",
"Young", "Sexy", "Classic", "Romantic", "Folk" and "Sport".

Category 4: Let $\mathrm{C}=\{\mathrm{c} 1 \ldots \mathrm{ck}\}$ be a set of $\mathrm{k}$ visual images $(\mathrm{k}=6)$.

Let $\mathrm{N}$ be the total number of input variables (Categories 2, 3, and 4). We have $\mathrm{N}=\mathrm{m}+\mathrm{n}+\mathrm{k}$ because, for each specific consumer, her body shape (all the body shapes correspond to $\mathrm{m}$ inputs), and desired style keyword (all the style keywords correspond to $\mathrm{n}$ inputs) and visual image (all the visual images correspond to $\mathrm{k}$ inputs) constitute the complete consumer profile.

Category 5: Let $\mathrm{G}=\{\mathrm{g} 1, \ldots, \mathrm{gh}\}$ be a set of $\mathrm{h}$ fitting levels ( $\mathrm{h}=5)$, including "fitting-loose", "fitting-a little loose", "fitting-moderate", "fitting-a little tight" and "fitting-tight".

Category 6: Let $\mathrm{DW}=\{\mathrm{dw} 1, \ldots, \mathrm{dwx}\}$ be a set of $\mathrm{x}$ details of waist $(\mathrm{x}=3)$, including "waist-high", "waist-moderate", "waist-low".

Category 7: Let $D F=\{d f 1, \ldots, d f y\}$ be a set of $y$ details of leg opening $(\mathrm{y}=3)$, including "Bell-bottom", "Regular", "Pencil Pants".

Category 8: Let $\mathrm{DO}=\{\mathrm{do} 1, \ldots, \mathrm{doz}\}$ be a set of $\mathrm{z}$ details of ornamentals $(\mathrm{z}=2)$, including "Ornamentalmore", "Ornamental-little".

The above $M$ variables (Categories 5, 6, 7, 8) are generally used by garment designers and shoppers for describing jeans according to their experience. Therefore, these variables constitute the profile of a recommended product with $\mathrm{M}=\mathrm{h}+\mathrm{x}+\mathrm{y}+\mathrm{z}$.

Category 9: Let $\mathrm{I}=\{\mathrm{I} 1, \ldots, \mathrm{Iq}\}$ be a set of q weights $(\mathrm{q}=3)$ corresponding to the three input parts BS, S and C. They are obtained by human evaluations using the FAHP method.

Category 10: Let KBBS be the knowledge base obtained from the Experiments 1, describing the relations between BS (body shapes) and E (normalized evaluation criteria). It is expressed by a $(\mathrm{m} \times \mathrm{p})$-dimensional matrix.

Category 11: Let KBS be the knowledge base obtained from the Experiments 2, describing the relations between $\mathrm{S}$ (style keywords) and $\mathrm{E}$. It is expressed by a $(\mathrm{n} \times \mathrm{p})$-dimensional matrix.

Category 12: Let $\mathrm{KBC}$ be the knowledge base obtained from the Experiments 3, describing the relations between $\mathrm{C}$ (visual images) and $\mathrm{E}$. It is expressed by a $(\mathrm{k} \times \mathrm{p})$-dimensional matrix.

Category 13: Let KBG be the knowledge base obtained from the Experiments 4, describing the 
relations between $\mathrm{E}$ and $\mathrm{G}$ (garment fitting levels). It is expressed by a $(\mathrm{p} \times \mathrm{h})$-dimensional matrix.

Category 14: Let KBDW be the knowledge base obtained from the Experiments 4, describing the relations between E and DW (details of waist). It is expressed by a $(\mathrm{p} \times \mathrm{x})$-dimensional matrix.

Category 15: Let KBDF be the knowledge base obtained from the Experiments 4, describing the relations between $\mathrm{E}$ and DF (details of leg opening). It is expressed by a $(\mathrm{p} \times \mathrm{y})$-dimensional matrix.

Category 16: Let KBDO be the knowledge base obtained from the Experiments 4, describing the relations between $\mathrm{E}$ and DO (details of ornamentals). It is expressed by a $(\mathrm{p} \times \mathrm{z})$-dimensional matrix.

Category 17: Let KB1 be the knowledge base obtained from the Experiments 1, 2, 3, describing the relations between the $\mathrm{N}$ input variables (consumer profiles) and $\mathrm{E}$ (normalized evaluation criteria). It is the combination of the matrices in Categories 11, 12 and 13 and then expressed by a $(\mathrm{N} \times \mathrm{p})$-dimensional matrix. Based on the values of the consumer profile-related matrices $\mathrm{KB}_{\mathrm{BS}} \mathrm{KB}_{\mathrm{S}}$ and $\mathrm{KB}_{\mathrm{C}}$, we obtain the matrix

$$
\mathrm{KB}_{1}=\left[\begin{array}{c}
\mathrm{KB}_{\mathrm{BS}} \\
\mathrm{KB}_{\mathrm{S}} \\
\mathrm{KB}_{\mathrm{C}}
\end{array}\right] .
$$

Category 18: Let KB2 be the knowledge base obtained from the Experiments 4 describing the relations between $\mathrm{E}$ and the $\mathrm{M}$ output variables (product profiles). It is the combination of the matrices in Categories 14, 15, 16 and 17, and then expressed by a $(p \times M)$-dimensional matrix. From the matrices of $\mathrm{KB}_{G}$, $\mathrm{KB}_{\mathrm{DW}}, \mathrm{KB}_{\mathrm{DF}}$ and $\mathrm{KB}_{\mathrm{DO}}$, we obtain the matrix $\mathrm{KB}_{2}=\left[\mathrm{KB}_{\mathrm{G}} \mathrm{KB}_{\mathrm{DW}} \mathrm{KB}_{\mathrm{DF}} \mathrm{KB}_{\mathrm{DO}}\right]$.

By performing a composition operation between the matrices $\mathrm{KB} 1$ and $\mathrm{KB} 2$, we obtain the overall knowledge base $\mathrm{KB}$ with $\mathrm{KB}=\mathrm{KB} 1{ }^{\circ} \mathrm{KB} 2 . \mathrm{KB}$ is a $(\mathrm{N} \times \mathrm{M})$-dimensional matrix, characterizing the relations between N-dimensional consumer profiles and M-dimensional product profiles.

Category 19: Let CP be a profile of a specific consumer including her body shape and desired style keywords and visual images. It is expressed by a $\mathrm{N}$-dimensional weighted vector denoted as

$\mathrm{CP}=\left(\mathrm{I}_{1} \times \mathrm{bs} 1, \ldots, \mathrm{I}_{1} \times \mathrm{bsm}, \quad \mathrm{I}_{2} \times \mathrm{s} 1, \ldots, \mathrm{I}_{2} \times \mathrm{sn}\right.$, $\left.\mathrm{I}_{3} \times \mathrm{c} 1, \ldots, \mathrm{I}_{3} \times \mathrm{ck}\right)$.

Category 20: Let $\mathrm{Y}$ be the desired product profile which is calculated by performing the composition operation of $\mathrm{CP}$ and $\mathrm{KB}$. $\mathrm{Y}$ represents the desired product profile by $\mathrm{CP}$ and is expressed by an M-dimensional vector.

Category 21: Let $\{\mathrm{y} 1, \ldots, \mathrm{yr}\}$ be a set of $\mathrm{r}$ garment products existing in the e-shop. They can also be expressed by $\mathrm{r}$ M-dimensional vectors.

\subsection{The Structure of the knowledge base}

The knowledge-based recommendation module aims at proposing the most suitable garment to a specific consumer according to her specific body shape and desired fashion image and style keywords by learning from the evaluation data.

The detailed procedure for implementation of the knowledge-based recommendation module is given below (see Figure 1). Two knowledge bases are built in this module. Knowledge Base 1 characterizes the relations between a consumer profile and 8 normalized sensory criteria.

Knowledge Base 2 characterizes the relations between a garment profile and the same sensory criteria and both knowledge bases are built from the social network and sensory evaluation of experts. After calculating the composition of the Knowledge Base 1 and the Knowledge Base 2, a jean can be recommended. Moreover, the consumer can visualize the virtual try on effect using the CLO3D.

\subsection{Details of the experiments}

More details about the consumer profiles are given below.

i. Body data, including four basic body measurements: b1: Stature, b2: Chest Circumstance, b3: Waist Circumstance, b4: weight.

ii. Style keywords, including 8 emotional keywords used for describing desired fashion styles of garments. These style keywords are semantically different from the 8 normalized sensory evaluation criteria which are linking consumer and product profiles. The style keywords are more emotional, variable with fashion trends and close to consumers' expectations on products while the 8 normalized sensory criteria are relatively neutral, stable and mastered by shopping experts for describing the nature of products. The most used style words include: w1: Elegant, w2: Feminine, w3: Young, 
w4: Sexy, w5: Classic, w6: Romantic, w7: Folk, w8: Sport $(n=8)$. The process of selecting the style keywords is given as follows. First, twenty various style keywords describing female jeans are summarized from the Internet and fashion magazines. Then each fashion expert evaluates the relevancy of each style keyword to female jeans with the help of the corresponding explanation by giving an evaluation score from 1 to 10 . The final 8 style keywords are determined by selecting those corresponding to the 8 highest averaged evaluation scores.

iii. Visual images: the consumer should choose a pair of favorite jeans from 6 pictures: $1, \mathrm{p} 2, \mathrm{p} 3, \mathrm{p} 4, \mathrm{p} 5$ and $\mathrm{p} 6(\mathrm{k}=6)$. During a purchasing activity, it is possible that consumers do not master appropriate verbal expressions describing their expectations. Therefore, we perform a series of evaluations so that the selected consumers of the target population select the most relevant images for extracting their real requirements.

\section{Body Data}

The Chinese Standard Human Body Database (female) is available for us. The statures of these samples are arranged from $145 \mathrm{~cm}$ to $175 \mathrm{~cm}$ with a step length of 5 $\mathrm{cm}$. According to this database, four standard body styles (Y, A, B, C) are defined from the difference of chest circumstance and waist circumstance.

In this database, the shortest sample is $145 \mathrm{~cm}$ and the tallest $175 \mathrm{~cm}$ and between them the other samples are arranged with an interval of $5 \mathrm{~cm}$. Generally, a number of common rules for human body sizing can be extracted.

\section{Fuzzy description of height}

Normally, we use two indices to describe a human body shape, namely height and fat-thin. However, how to evaluate tall-low and fat-thin is vague. It is for this reason that we use fuzzy sets to express human body shapes.

Based on the garment designer's knowledge, tall-low can be expressed by b1, and fat-thin can be expressed by b1 \& b4.

According to the previous database of human body shapes, $160 \mathrm{~A}$ is generally taken as the standard body shape for Chinese female population, and $145 \mathrm{~cm}$ and $175 \mathrm{~cm}$ can be considered as the lower and upper bounds of the whole population. We can describe the tall-low as five levels: X1: short, X2: a little short, X3: middle, X4: a little tall, X5: tall.

The fuzzy function and rules are obtained by uniformly dividing the whole range of the body data in height into 5 classes or 5 fuzzy values, denoted as X1, $\mathrm{X} 2, \mathrm{X} 3, \mathrm{X} 4, \mathrm{X} 5$.

\section{Fuzzy description of fat and thin}

BMI (Body Mass Index = Weight in Kilograms / (Height in Meters $x$ Height in Meters)) is a measurement of body fat based on height and weight and applied to both men and women from 18 to 65 years.

The WTO regards a BMI of less than 18.5 as underweight and may indicate malnutrition, an eating disorder, or other health problems, while a BMI equal to or greater than 25 is considered overweight and above 30 is considered obese. The range of BMI values is valid only as statistical categories.

Based on the information, we can define fat-thin as four levels: Y1: underweight Y2: normal, Y3: overweight, Y4: obese. Therefore, the whole body shape can be described using one of $5 \times 4$ fuzzy values $(\mathrm{m}=20)$.

The proposed recommendation system permits to generate two outputs (product profile):

\section{Fitting of a Garment Style}

The fitting of a garment style can be perceived using a number of frequently used emotional keywords. In this paper, we just extract those from the H\&M shopping guide: Skinny, Slim, Straight, Loose. However, for different brands, there are different fashion rules on garment style fitting. To solve this problem we make a unified neutral description of garment fitting by defining a fuzzy variable of five values: tight, a little tight, moderate, a little loose, loose $(\mathrm{h}=5)$.

\section{Details}

Based on the design or shopping experts' knowledge, we take three details: waist, foot opening levels and ornamental. And we formalize them according to the same principle as the garment style fitting. The three defined fuzzy variables describing the details are given below.
i. Low-waist, Regular-waist, High-waist $(x=3)$
ii. Pencil Pants, Regular, Bell-bottom ( $\mathrm{y}=3$ )
iii. Ornamental, without ornamental $(\mathrm{z}=2)$ 
We have four sensory experiments for collecting human perception data. Experiment 1,2 and 3 aim at finding out the relations between body types, style keywords, visual images and the 8 evaluation criteria. Experiment 4 finds out the relations between the design styles and the same 8 evaluation criteria. The combination of Experiment 1,2 and 3 permits to generate Knowledge Base 1 while the results of Experiment 4 are used to form Knowledge Base 2. The relation is given below (see Figure 2).

\section{Standard evaluation criteria}

These 8 normalized pairs of descriptors constitute the intermediate layer connecting both the inputs (consumer's profile) and outputs (recommended product). They are as follows: 1.usual-unique, 2.complicated-simple, 3.informal-formal, 4.fashionabletraditional, 5.Lovely-mature, 6.relaxed-serious, 7.citied -rural, 8.Intellectual-spirit. The principle of their selection is briefly presented below. We first collect the exhaustive expressions describing garments given by all the trained evaluators (shopping experts) without considering whether they make sense or not. The words collected previously are screened through a discussion among the evaluators. The collected words are further refined by comparison with the frequently used expressions describing human emotions on consumer goods. 17 Finally, 8 pairs of words are determined as the standardized evaluation criteria in our study for describing both consumers' expectations and products.

Each pair of the descriptors is described using five scores such as: VI (very informal), I (informal), M (medium), F (formal), VF (very formal).

For a group of evaluators, their evaluation scores for any criterion $\mathrm{x}$ are not the same and then we use a fuzzy value to express the distribution of all evaluation scores, i.e. $x \rightarrow\left(v^{V I}(x) v^{I}(x) v^{M}(x) v^{F}(x) v^{V F}(x)\right) T$, where $v_{i}$ means the proportion of the number of votes for the score $\mathrm{j}$ to the total number of evaluators.

\subsection{A real case}

The proposed recommendation module is validated by its application to a real jeans recommendation case for a specific female consumer Y01. The consumer profile of $\mathrm{Y} 01$ is composed of the following input data: $\mathrm{kg}$.

Body data: $\mathrm{b} 1=163 \mathrm{~cm} \mathrm{b2}=104 \mathrm{~cm} \mathrm{b3}=92 \mathrm{~cm} \mathrm{~b} 4=50$

Style keyword: w1 (elegant).
Visual image: $p 1$.

According to her body data, we identify the corresponding normalized body size: 165B. The consumer profile $\mathrm{CP}$ is: $(0,0,0,0,0,0,0,0.31,0.16,0,0,0$, $0.005,0.005,0,0,0,0,0,0,0.31,0,0,0,0,0,0,0,0.21,0,0,0,0,0)$.

According to the fuzzy composition operation, we obtain the product profile desired by Y01 as follows.

$$
\begin{aligned}
& \mathrm{Y}=\mathrm{CP}^{\circ} \mathrm{KB}= \\
& (0.3,0.3,0.4,0.8,0.4,0.3,0.8,0.6,0.4,0.7,0.6,0.3,0.7) .
\end{aligned}
$$

In a specific garment e-shop, we have 3 products, whose product profiles are provided by the marketing experts. We should find out which product is the most suitable for the consumer Y01. These product profiles are given below.

$$
\begin{aligned}
& \mathrm{y} 1=(0.5,0.9,0.2,0.4,0.4,0.3,0.3,0.5,0.9,0.5,0.4,0.3,0.8) . \\
& \mathrm{y} 2=(0.3,0.4,0.6,0.8,0.5,0.2,0.7,0.6,0.4,0.7,0.5,0.3,0.7) . \\
& \mathrm{y} 3=(0.8,0.3,0.8,0.6,0.2,0.7,0.1,0.3,0.4,0.5,0.6,0.7,0.2) .
\end{aligned}
$$

We calculate their similarity degrees with $\mathrm{Y}$ by using the Hamming similarity degree. We have

$$
\begin{aligned}
& \mathrm{N}_{1}(\mathrm{Y}, \mathrm{y} 1)=0.77 \\
& \mathrm{~N}_{1}(\mathrm{Y}, \mathrm{y} 2)=0.95 \\
& \mathrm{~N}_{1}(\mathrm{Y}, \mathrm{y} 3)=0.71
\end{aligned}
$$

We find that $y 2$ (see Figure 3) corresponds to the biggest similarity degree and then recommend it to the consumer Y01. If this consumer is not satisfied with the result, we can adjust the knowledge base based on the evaluation of the selected product by this consumer (the details are giving in knowledge updating module). Otherwise, this module stops.

\section{The Knowledge Updating Module}

In the previous sections, we have proposed knowledge-based recommendation modules to recommend product profiles for each consumer according to her general or personalized requirements. However, this recommendation module has the two major drawbacks.

i. They cannot guarantee the satisfaction of recommendations results by consumers.

ii. The structures of the modules and the knowledge base involved are not flexible and cannot be adapted to various scenarios. One example is that the consumer must choose some existing style keywords 
from a fixed list of "Elegant", "Feminine", "Young", "Sexy", "Classic", "Romantic", "Folk" and "Sport", instead of proposing new keywords.

In this context, we propose a new module, i.e. the Knowledge Updating Module, aiming at overcoming these drawbacks by introducing a mechanism of feedback. The main principle is described below (See Figure 4).If the consumer is not satisfied with the recommended result provided by the Knowledge-Based Module, she/he will quantitatively identify the unsatisfied part of the recommended garment. The Knowledge Updating Module will adjust the knowledge base and recommend another product to this consumer. This procedure is repeated until the full satisfaction of the recommended result by the consumer. The knowledge base can be progressively improved by integrating the consumer's evaluation results of a series of recommended products. Due to its strong self-learning ability, this module can progressively improve the quality of the whole garment recommendation system by introducing a large number of consumers' evaluations on recommended products.

It is composed of the following functional blocks: 1) Virtual try-on and consumer's evaluation, 2) Knowledge base updating 1, 3) Knowledge base updating 2.

\subsection{Virtual try-on and evaluation}

Having received the recommended product profile, we use the Clo3D garment CAD software for visualizing its fitting effects on the virtual body shape of the consumer. One example of virtual female jeans try-on is given in Figure 5.

Based on the visual effects of the virtual product generated from the recommended profile in the CLO3D environment, the consumer gives her evaluation scores on the difference between the recommended and desired product profile. The evaluation procedure, including the steps of training, evaluation scale definition and adjustment of desired product profile, is described below.

\section{i. Training}

Before the evaluation, this consumer should carefully read the guide instructions for 5 minutes in order to understand the evaluation method to be used and related to garment styles and details. We use the following four descriptors in this evaluation procedure, i.e. garment fitting, detail of waist, details of leg opening and details of ornamental. During the training, the consumer should understand the meaning of each evaluation descriptor.

Next, the consumer will take about 10 minutes for evaluating the two visual images of the recommended product, i.e. the front image (see in Figure 5-a) and the back image (see in Figure 5-b).

ii. Scale definition

The method of semantic differential scale is used in our evaluation procedure for evaluating the recommended product related to the four descriptors. The evaluation scores take value from the normalized set $\{-2,-1,0,1,2\}$ for all the four descriptors. These scores represent different meanings for different descriptors. For example, for "fitting level", these five scores correspond to the linguistic values \{"tight", "a little tight", "perfect", "a little loose", "loose"\} respectively.

iii. Adjustment of the desired product profile based on the consumer evaluation.

The new desired product profile $\mathrm{Y}^{*}$ can be defined by $\mathrm{Y}^{*}=\left(\mathrm{g}_{1}{ }^{*}, \cdots, \mathrm{g}_{\mathrm{h}}{ }^{*}, \mathrm{dw}_{1}{ }^{*}, \cdots, \mathrm{dw}_{\mathrm{x}}{ }^{*}, \mathrm{df}_{1}{ }^{*}, \cdots, \mathrm{df}_{\mathrm{y}}{ }^{*}, \mathrm{do}_{1}{ }^{*}, \cdots, \mathrm{do}_{\mathrm{z}}{ }^{*}\right)$

$\mathrm{Y}^{*}$ is an M-dimensional vector where $\mathrm{M}=\mathrm{h}+\mathrm{x}+\mathrm{y}+\mathrm{z}$, $h$ garment fitting levels $(h=5), x$ details of waist $(x=3), y$ details of leg opening $(y=3)$, and $z$ details of ornamentals $(\mathrm{z}=2)$.

In the following tables (Tables 1, 2, 3, 4), we define 4 rules characterizing the deviations $\Delta \mathrm{Y}$ from the former product profile $\mathrm{Y}$ (calculated from the composition of the knowledge base $\mathrm{KB}$ and the consumer profile $\mathrm{CP}$ ) to $\mathrm{Y}^{*}$ according to the consumer evaluation scores. We have $\mathrm{Y}^{*}=\mathrm{Y}+\Delta \mathrm{Y}$. The rules of Table 1 are defined according to the following design principles given by experienced designers: 1) if the garment fitting is too tight, then we should adjust it with bigger deviation values over all the fitting levels (rough adjustment), 2) if the fitting is a little tight, then we should adjust it with smaller deviation values (fine adjustment), 3) the same principle is applied to "loose" and "a little loose".

Using the above rules, we can obtain a new consumer desired product profile $\left(\mathrm{Y}^{*}\right)$ based on the consumer evaluation scores.

For example, during the evaluation of a recommended jeans, if the consumer considers that the garment fitting level is a little tight (“-1") and the other three descriptors are perfect ("0"), then we obtain the new product profile $\mathrm{Y}^{*}$ with $\mathrm{g}_{1}{ }^{*}=\mathrm{g}_{1}+0.1 \times \mathrm{g}_{1}$, 
$\mathrm{g}_{2}{ }^{*}=\mathrm{g}_{2}+0.05 \times \mathrm{g}_{2}, \quad \mathrm{~g}_{3}{ }^{*}=\mathrm{g}_{3}, \mathrm{~g}_{4}{ }^{*}=\mathrm{g}_{4}-0.05 \times$ $\mathrm{g}_{4}$ and $\mathrm{g}_{5}{ }^{*}=\mathrm{g}_{5}-0.1 \times \mathrm{g}_{5}$, the other parts of $\mathrm{Y}$ are not changed. (based on Rule 1).

\subsection{Knowledge base updating 1}

In the previous section, we calculated the new desired product profile $\mathrm{Y}^{*}$ from the consumer evaluation. For the same Consumer Profile (CP), we can obtain a temporary Knowledge Base $\mathrm{KB}^{*}$ by using the relation $\mathrm{Y}^{*}=\mathrm{CP}^{\circ} \mathrm{KB}^{*}$.

$\mathrm{KB}^{*}$ is a $(\mathrm{N} \times \mathrm{M})$-dimensional matrix taking into account the feedback information given by the consumer. In general, $\mathrm{KB}^{*}$ is just a slight modification from $\mathrm{KB}$ because $\mathrm{Y}^{*}$ is just a slight adjustment of $\mathrm{Y}$. Based on $\mathrm{Y}^{*}$, we will further adjust the permanent knowledge base $\mathrm{KB}$ so that it takes into account both the already identified relations between consumer profiles and product profiles and the new consumer evaluation results. The changes of KB should be slight and strictly controlled so that they cannot destroy the existing relations between garment styles, normalized evaluation criteria and consumer profiles. The generation of $\mathrm{KB}^{*}$ is performed as follows.

First, we let $\mathrm{KB}^{*}:=\mathrm{KB}$.

For facilitating the following discussion, we denote the elements of the consumer profile $\mathrm{CP}$, the desired product profile $\mathrm{Y}^{*}$ and the updated knowledge base $\mathrm{KB}^{*}$ as follows.

$$
\begin{gathered}
\mathrm{CP}=\left(\mathrm{cp}_{1}, \mathrm{cp}_{2}, \ldots, \mathrm{cp}_{\mathrm{N}}\right) \\
\mathrm{Y}^{*}=\left(\mathrm{y}_{1}, \mathrm{y}_{2}, \ldots, \mathrm{y}_{\mathrm{M}}\right) \\
\mathrm{KB}^{*}=\left[\begin{array}{cccc}
\mathrm{kb}_{11} & \mathrm{~kb}_{12} & \cdots & \mathrm{kb}_{1 \mathrm{M}} \\
\mathrm{kb}_{21} & \mathrm{~kb}_{22} & \cdots & \mathrm{kb}_{2 \mathrm{M}} \\
\vdots & \vdots & \ddots & \vdots \\
\mathrm{kb}_{\mathrm{N} 1} & \mathrm{~kb}_{\mathrm{N} 2} & \cdots & \mathrm{kb}_{\mathrm{NM}}
\end{array}\right]
\end{gathered}
$$

According to the composition relation between them, we have

$$
\begin{aligned}
\mathrm{y}_{\mathrm{j}}=\left(\mathrm{cp}_{1} \wedge \mathrm{kb}_{1 \mathrm{j}}\right) \vee\left(\mathrm{cp}_{2} \wedge \mathrm{kb}_{2 \mathrm{j}}\right) \ldots \\
\vee\left(\mathrm{cp}_{\mathrm{N}} \wedge \mathrm{kb}_{\mathrm{Nj}}\right) \mathrm{j} \in\{1,2, \ldots, \mathrm{M}\}
\end{aligned}
$$

From Eq. (1), (2), (3) and (4), there are two situations:

i. $y_{j} \leq \max _{1 \leq i \leq N}\left\{c_{i}\right\}$ for any $j \in\{1,2, \ldots, M\}$. In this situation, we set up two rules for assigning values of $\mathrm{KB}^{*}$ :
Rule 1: if $\exists \mathrm{cp}_{\mathrm{i}}$ s.t. $\mathrm{y}_{\mathrm{j}}=\mathrm{cp}_{\mathrm{i}}$ Then $\mathrm{kb}_{\mathrm{ij}}:=\mathrm{cp}_{\mathrm{i}}$

Rule 2: If we have $y_{j} \neq c p_{i}$ for all $i \in\{1,2, \ldots, N\}$, we separate the set of $\left\{\mathrm{cp}_{\mathrm{i}}\right\}$ into two parts by using the threshold $y_{j}$, i.e. $c p_{i_{1}}, c_{i_{2}}, \ldots, c p_{i_{r}}<y_{j}$, $\mathrm{cp}_{\mathrm{i}_{\mathrm{r}+1}}, \mathrm{cp}_{\mathrm{i}_{\mathrm{r}+2}}, \ldots, \mathrm{cp}_{\mathrm{i}_{\mathrm{N}}}>\mathrm{y}_{\mathrm{j}}$.

Then we define $k b_{i_{k} j}:=y_{j}$ for $k=r+1, \ldots, N$. Apart from the above changes, the remaining elements of $\mathrm{KB}^{*}$ maintain the same values as those of $\mathrm{KB}$.

ii. $\mathbf{y}_{\mathbf{j}}>\max _{1 \leq \mathrm{i} \leq \mathrm{N}}\left\{\mathbf{c p}_{\mathbf{i}}\right\}$ for any $\mathbf{j} \in\{1,2, \ldots, \mathbf{M}\}$

There is no solution for the equation $\mathrm{Y}^{*}=\mathrm{CP}{ }^{\circ} \mathrm{KB}^{*}$. In this case, we do not need to change the knowledge base $\mathrm{KB}$ but just to recommend a new product profile by finding the biggest similarity degree of $\mathrm{Y}^{*}$ related to the existing products in the shop based on the Hamming similarity degree.

If some changes in $\mathrm{KB}^{*}$ related to $\mathrm{KB}$ really exist, we need to update the permanent knowledge base $\mathrm{KB}$ by taking into account the values of $\mathrm{KB}^{*}$ (the consumer feedback information), obtained from the previous Rule 1 and Rule 2. We proposed the following updating formula:

$\mathrm{KB}:=\mathrm{KB} \times(1-\omega)+\mathrm{KB}^{*} \times \omega \omega \in[0,1] \omega$ is a small constant controlling the slight rate of change. Its value can be defined according to the expert experience.

Using these rules, we can adjust all elements of $\mathrm{KB}$ to a new knowledge base. The more $\mathrm{KB}$ is used, the more knowledge can be learned.

Next, based on the updated knowledge base KB, we obtain a new product profile desired by calculating $\mathrm{Y}=$ $\mathrm{CP}^{\circ} \mathrm{KB}$ again. This procedure is repeated until the consumer is satisfied with the recommended product.

Also, in order to avoid any deadlock in this procedure and increase the efficiency of recommendation, we just apply a rule prohibiting all already recommended products to be proposed for a second time.

\subsection{Knowledge base updating 2}

Apart from the updating of relations between consumer profiles and product profiles caused by consumer evaluations, we also propose to update the knowledge base caused by changes of basic elements in the consumer profile. In fact, as fashion trends vary very quickly with society, style keywords and visual images describing fashion products are generally changed after 
a time period. Some old keywords and visual images will disappear and some new ones will be introduced.

In this situation, we wish to update and extend the set of 8 style keywords and 6 visual images, previously used in the definition of the consumer profile, in order to follow evolutionary fashion requirements of consumers. With updating of the knowledge base KB caused by the new consumer profile, the proposed recommendation system will become more open.

In this knowledge updating procedure, we consider the replacement of one style keyword (visual image) by a new one and insertion of one new style keyword (visual image). Two cases will be discussed below.

The new keyword (visual image) is independent of all the existing ones

For example, the style keywords "Sport" becomes less fashioned and it is changed into "Wild", which is independent of all the existing keywords.

For replacing one style keyword by a new independent one, we should perform Sensory Experiment 2 with a number of trainees for identifying the relations of the new keyword with the 8 normalized evaluation criteria $\{\mathrm{e} 1, \ldots, \mathrm{e} 8\}$. The new data on these relations are then introduced to the matrix $\mathrm{KB}_{\mathrm{S}}$ in order to replace the row corresponding to the removed style keyword.

In this case, the matrix KBS is transformed into the matrix $\mathrm{KB}_{\mathrm{S}}^{*}$, which is also is a $(\mathrm{n} \times \mathrm{p})$-dimensional matrix.

The matrix of KB1 can be adjusted by combing $\mathrm{KB}_{\mathrm{BS}}$ (unchanged relations on body shapes), $\mathrm{KB}_{\mathrm{S}}^{*}$ (relations on style keywords with one replacement), and $\mathrm{KB}_{\mathrm{C}}$ (unchanged relations on visual images) together.

$$
\mathrm{KB}_{1}:=\left[\begin{array}{c}
\mathrm{KB}_{\mathrm{BS}} \\
\mathrm{KB}_{\mathrm{S}}^{*} \\
\mathrm{~KB}_{\mathrm{C}}
\end{array}\right] .
$$

Based on the new $(\mathrm{N} \times \mathrm{p})$-dimensional matrix $\mathrm{KB} 1$, we obtain the new knowledge base KB.

For replacing one visual image by a new one, the procedure is the same. For inserting a new keyword or visual image, the procedure is also the same but $\mathrm{KB} 1$ will be extended to a $((\mathrm{N}+1) \times \mathrm{p})$-dimensional matrix and the former relations in $\mathrm{KB} 1$ are unchanged.

The new keyword (visual image) is a combination of all the existing ones
For example, the new keyword "faddish" can be considered as a combination of all the existing keywords and will be added to the system.

In this case, we do not need to perform any new sensory experiment but just identify this combination in order to express the relations of the new style keyword $\mathrm{sn}+1$ using the existing ones, i.e. $\{\mathrm{s} 1, \ldots, \mathrm{sn}\}$. The weights of this combination have been computed from the weighted combination of these style keywords using the Fuzzy AHP method, like in Section 3.3. In this situation, we have

$$
s_{n+1}=t_{1} \times s_{1}+t_{2} \times s_{2}+\cdots+t_{n} \times s_{n}
$$

where $t 1, \ldots$, tn are the weights of the existing keywords $s 1, \ldots$, sn respectively, provided by experts.

Based on this identified combination, we can update KBs by replacing the corresponding row or inserting a new row. The same procedure can be applied to replacement of one visual image or insertion of one new visual image.

\section{Validation of the Recommendation System}

In this section, the validation of these modules is realized from both individual shopping cases and statistical results for a target population. In this study, 60 consumers randomly selected from the target population (Chinese women aged from18 to 25) are invited to make concerned evaluations and virtual garment try-on. A garment e-shop is created with 9 existing garments and 2 successful cases.

The profiles of these 9 products are provided by the marketing experts as follows.

$$
\begin{aligned}
& \mathrm{y} 1=(0.5,0.9,0.2,0.4,0.4,0.3,0.3,0.5,0.9,0.5,0.4,0.3,0.8) . \\
& \mathrm{y} 2=(0.3,0.4,0.6,0.8,0.5,0.2,0.7,0.6,0.4,0.7,0.5,0.3,0.7) \text {. } \\
& \mathrm{y} 3=(0.8,0.3,0.8,0.6,0.2,0.7,0.1,0.3,0.4,0.5,0.6,0.7,0.2) \text {. } \\
& \mathrm{y} 4=(0.5,0.7,0.2,0.4,0.4,0.3,0.3,0.5,0.9,0.5,0.4,0.3,0.8) \text {. } \\
& \mathrm{y} 5=(0.2,0.3,0.5,0.6,0.9,0.1,0.4,0.9,0.4,0.3,0.8,0.4,0.6) \text {. } \\
& \mathrm{y} 6=(0.5,0.5,0.7,0.6,0.3,0.3,0.8,0.6,0.4,0.7,0.6,0.3,0.7) . \\
& \mathrm{y} 7=(0.8,0.7,0.4,0.4,0.3,0.1,0.2,0.4,0.9,0.5,0.2,0.8,0.4) \text {. } \\
& \mathrm{y} 8=(0.8,0.7,0.4,0.2,0.3,0.7,0.2,0.4,0.3,0.5,0.7,0.8,0.4) \text {. } \\
& \mathrm{y} 9=(0.1,0.3,0.4,0.7,0.5,0.1,0.3,0.8,0.5,0.7,0.6,0.1,0.7) .
\end{aligned}
$$

\section{Scenario 1:}

A consumer is visiting the e-shop. Her profile $\mathrm{CP}$ is $(0,0,0,0,0,0,0,0.31,0.16,0,0,0,0.005,0.005,0,0,0,0,0,0$, $0.31,0,0,0,0,0,0,0, \quad 0.21,0,0,0,0,0) . \quad$ The

Knowledge-based Recommendation Module is applied to this consumer. By using the composition operation 
between $\mathrm{CP}$ and the knowledge base $\mathrm{KB}$, we obtain the desired product profile:

$$
\mathrm{Y}=(0.3,0.3,0.4,0.8,0.4,0.3,0.8,0.6,0.4,0.7,0.6,0.3,0.7)
$$

Next, we select y2 from the e-shop the product whose similarity degree related to the expected product profile $\mathrm{Y}$ is the biggest $(=0.95)$. The corresponding virtual try-on is given in Figure 6. However, the consumer is not satisfied with this product.

After the evaluation of the recommended jeans, this consumer considers that the garment fitting level is a little tight ("- 1 ") and the other three descriptors are perfect ("0"). The Knowledge Updating Module will adjust the knowledge base $\mathrm{KB}$ for this consumer and another product $\mathrm{y} 6$ of the e-shop will be recommended by using the new knowledge base KB. The difference between the new $\mathrm{KB}$ and the old $\mathrm{KB}$ is very tiny, since the adjustment of product profile $\mathrm{Y}$ is slight.

The virtual try-on of this new product is given in Figure 7 , we can see that a visually looser product is recommended to this consumer, and this consumer is satisfied with it, showing that the Knowledge Updating Module can really improve the quality of recommendation.

Next, we carry out the validation using the statistical results with all the 60 selected consumers.

\section{Scenario 2:}

In the experiment, we invite 20 of the 60 consumers of Group 2 to use this module only without providing any feedback for updating the knowledge base (Scenario $2.1)$. Only 6 of them obtained satisfying results. Next, 20 of them are invited to use this module and provide their feedback for updating the knowledge base if they are not satisfied with the recommended results (Scenario 2.2). The maximal number of recommendations is set to 4 . We consider that the system fails if the consumer is not satisfied with 4 recommendations results. In this case, 18 of them obtained satisfying results and the averaged number of applying the Knowledge-Based Recommendation Module is 2.3 times. The last 20 persons are invited to use this module by adding (or changing) a new style keyword or a new fashion image (Scenario 2.3). 13 of them obtained satisfying results and the averaged number of applying the Knowledge-Based Recommendation Module is 3.1 times. The corresponding results are shown in Table 5 .
From Table 5, we can notice that, in Scenario 2, only $30 \%$ of consumers are satisfied with the recommendation results if the consumers' feedback and knowledge updating does not exist, while $90 \%$ of consumers can be satisfied when we introduce the cycle of recommendation - consumer evaluation - feedback knowledge updating. Also, $65 \%$ of consumers can be satisfied if they are allowed to change a desired style keyword (fashion image) or add a new one when inputting their consumer profiles. These results show that the updating of the knowledge base with consumers' feedback and change of desired style keywords (fashion images) can effectively improve the success rate of product recommendations.

Moreover, the numbers of recommendations with these two kinds of updating are rather limited (2.3 for updating with consumers' feedback, 3.1 for updating with change of desired style keywords (fashion images)). In general, the performance of updating with consumers' feedback is better than that of updating with change of desired style keywords (fashion images).

\section{Conclusions and Future Work}

This garment recommendation system provides a powerful support for suggesting the most appropriate decision support on garment purchasing for various consumers in different demands. It enables to adjust the knowledge base according to the feedback information about consumers in order to improve the satisfaction degree of recommendation. The knowledge base can also be updated by changing or adding elements of the consumer profile. This module makes the whole recommendation system more flexible and more adapted to changes of fashion trends and consumers' perception and preferences.

Due to the time limitation, the current work are still far from being perfect. In future research, more effort should be dedicated to the following aspects:

i. The number of samples involved in the present $\cdot$ study is quite limited and the conclusions are drawn upon only one specific type of garment products (jeans). In practice, reliable recommendation results depend on a big and comprehensive experimental dataset. Therefore, in the future, in order to obtain more generalized and concrete information about the knowledge, it is 
imperative to integrate more types of samples with more diversified garment styles and details into the system.

ii. Self learning is realized by the current knowledge base, according to consumer feedback, the

\section{Reference}

1. Shih, H.-P. An empirical study on predicting user acceptance of e-shopping on the Web. Information \& Management, 2004. 41(3): pp. 351-368.

2. $\mathrm{Wu}, \mathrm{D}$., et al. A fuzzy preference tree-based recommender system for personalized business-tobusiness e-services. IEEE Transactions on Fuzzy Systems, 2015. 23(1): pp. 29-43.

3. Yoo, C.W., et al. The impact of interactivity of electronic word of mouth systems and E-Quality on decision support in the context of the e-marketplace. Information \& Management, 2015. 52(4): pp. 496-505.

4. Toledo, R.Y. and L. Martinez, Fuzzy Tools in Recommender Systems: A Survey. International Journal of Computational Intelligence Systems, 2017: pp. 776-803.

5. Schafer, J.B., et al. Collaborative filtering recommender systems, in The adaptive web. 2007, Springer. pp. 291-324.

6. Pazzani, M.J., A framework for collaborative, content-based and demographic filtering. Artificial Intelligence Review, 1999. 13(5-6): pp. 393-408.

7. Barranco, M.J., A Knowledge Based Recommender System with Multigranular Linguistic Information. International Journal of Computational Intelligence Systems, 2008. 1(3): pp. 225-236.

8. Kardan, A., et al. A novel approach to hybrid recommendation systems based on association rules mining for content recommendation in asynchronous discussion groups. Information Sciences, 2013. 219(219): pp. 93-110.

9. Schafer, J.B., et al. E-commerce recommendation applications, in Applications of Data Mining to Electronic Commerce. 2001, Springer. pp. 115-153. knowledge base can be automatically adjusted. However, the learning strategy of the knowledge base is still too simplified. In the future, more intelligent strategies can be introduced to make the knowledge base more accurate and intelligent.

10. Amatriain, X., Mining large streams of user data for personalized recommendations. ACM SIGKDD Explorations Newsletter, 2013. 14(2): pp. 37-48.

11. Zhu, Y., et al. Characterization of fashion themes using fuzzy techniques for designing new human centered products. International Journal of Computational Intelligence Systems, 2010. 3(4): pp. 452-460.

12. $\mathrm{Hu}, \mathrm{J}$, et al. A novel networked traffic parameter forecasting method based on Markov chain model. in IEEE International Conference on Systems, Man and Cybernetics. 2003:pp.3595-3600 vol.4.

13. Das, A., C. Mathieu, and D. Ricketts, Maximizing profit using recommender systems. arXiv preprint arXiv: 0908.3633, 2009:1-5.

14. Sutar, S.H. and A.H. Khade, Recommendation System for Outfit Selection (RSOS). arXiv preprint arXiv:1402.6692, 2014:1-6.

15. Ichimura, T. and I. Tachibana. Emotion orientated recommendation system for Hiroshima tourist by fuzzy Petri Net. in Computational Intelligence \& Applications (IWCIA), 2013 IEEE Sixth International Workshop on 2013:pp.796-801.

16. Zheng, D., et al. Emotion Chat: A Web Chatroom with Emotion Regulation for E-Learners. Physics Procedia, 2012. 25: pp. 763-770.

17. Schrödter, R., AM Munoz, G.-V. Civille und BT Carr: Sensory Evaluation in Quality Control. 240 Seiten, zahlr. Abb. und Tab. Van Nostrand Reinhold, New York 1992. Preis: 30,-US\$. Food/Nahrung, 1993. 37(6): pp. 619-619. 
Table 1. Rule 1: adjustment of the fitting levels.

\begin{tabular}{clllll}
\hline Deviation on fitting level & $\begin{array}{l}\text { tight } \\
(-2)\end{array}$ & $\begin{array}{l}\text { a little tight } \\
(-1)\end{array}$ & $\begin{array}{l}\text { perfect } \\
(0)\end{array}$ & $\begin{array}{l}\text { a little loose } \\
(1)\end{array}$ & $\begin{array}{l}\text { Loose } \\
(2)\end{array}$ \\
\hline$\Delta \mathrm{g}_{1}=\mathrm{g}_{1}^{*}-\mathrm{g}_{1}$ & $0.2 \times \mathrm{g}_{1}$ & $0.1 \times \mathrm{g}_{1}$ & 0 & $-0.1 \times \mathrm{g}_{1}$ & $-0.2 \times \mathrm{g}_{1}$ \\
$\Delta \mathrm{g}_{2}=\mathrm{g}_{2}^{*}-\mathrm{g}_{2}$ & $0.1 \times \mathrm{g}_{2}$ & $0.05 \times \mathrm{g}_{2}$ & 0 & $-0.05 \times \mathrm{g}_{2}$ & $-0.1 \times \mathrm{g}_{2}$ \\
$\Delta \mathrm{g}_{3}=\mathrm{g}_{3}^{*}-\mathrm{g}_{3}$ & 0 & 0 & 0 & 0 & 0 \\
$\Delta \mathrm{g}_{4}=\mathrm{g}_{4}^{*}-\mathrm{g}_{4}$ & $-0.1 \times \mathrm{g}_{4}$ & $-0.05 \times \mathrm{g}_{4}$ & 0 & $0.05 \times \mathrm{g}_{4}$ & $0.1 \times \mathrm{g}_{4}$ \\
$\Delta \mathrm{g}_{5}=\mathrm{g}_{5}^{*}-\mathrm{g}_{5}$ & $-0.2 \times \mathrm{g}_{5}$ & $-0.1 \times \mathrm{g}_{5}$ & 0 & $0.1 \times \mathrm{g}_{5}$ & $0.2 \times \mathrm{g}_{5}$ \\
\hline
\end{tabular}

Table 2. Rule 2: adjustment of the details of waist.

\begin{tabular}{cccccc}
\hline $\begin{array}{c}\text { Deviation on } \\
\text { detail of waist }\end{array}$ & $\begin{array}{c}\text { high } \\
(-2)\end{array}$ & $\begin{array}{c}\text { a little high } \\
(-1)\end{array}$ & $\begin{array}{c}\text { perfect } \\
(0)\end{array}$ & $\begin{array}{c}\text { a little low } \\
(1)\end{array}$ & $\begin{array}{c}\text { low } \\
(2)\end{array}$ \\
\hline$\Delta \mathrm{dw}_{1}=\mathrm{dw}_{1}^{*}-\mathrm{dw}_{1}$ & $-0.2 \times \mathrm{dw}_{1}$ & $-0.1 \times \mathrm{dw}_{1}$ & 0 & $0.1 \times \mathrm{dw}_{1}$ & $0.2 \times \mathrm{dw}_{1}$ \\
$\Delta \mathrm{dw}_{2}=\mathrm{dw}_{2}^{*}-\mathrm{dw}_{2}$ & 0 & 0 & 0 & 0 & 0 \\
$\Delta \mathrm{dw}_{3}=\mathrm{dw}_{3}^{*}-\mathrm{dw}_{3}$ & $0.2 \times \mathrm{dw}_{3}$ & $0.1 \times \mathrm{dw}_{3}$ & 0 & $-0.1 \times \mathrm{dw}_{3}$ & $-0.2 \times \mathrm{dw}_{3}$ \\
\hline
\end{tabular}

Table 3. Rule 3: Adjustment of the details of leg opening.

\begin{tabular}{cccccc}
\hline $\begin{array}{c}\text { Deviation on detail } \\
\text { of leg opening }\end{array}$ & $\begin{array}{c}\text { big } \\
(-2)\end{array}$ & $\begin{array}{c}\text { a little big } \\
(-1)\end{array}$ & $\begin{array}{c}\text { perfect } \\
(0)\end{array}$ & $\begin{array}{c}\text { a little small } \\
(1)\end{array}$ & $\begin{array}{c}\text { small } \\
(2)\end{array}$ \\
\hline$\Delta \mathrm{df}_{1}=\mathrm{df}_{1}^{*}-\mathrm{df}_{1}$ & $-0.2 \times \mathrm{df}_{1}$ & $-0.1 \times \mathrm{df}_{1}$ & 0 & $0.1 \times \mathrm{df}_{1}$ & $0.2 \times \mathrm{df}_{1}$ \\
$\Delta \mathrm{df}_{2}=\mathrm{df}_{2}^{*}-\mathrm{df}_{2}$ & 0 & 0 & 0 & 0 & 0 \\
$\Delta \mathrm{df}_{3}=\mathrm{df}_{3}^{*}-\mathrm{df}_{3}$ & $0.2 \times \mathrm{df}_{3}$ & $0.1 \times \mathrm{df}_{3}$ & 0 & $-0.1 \times \mathrm{df}_{3}$ & $-0.2 \times \mathrm{df}_{3}$ \\
\hline
\end{tabular}

Table 4. Rule 4: Adjustment of the details of ornamental.

\begin{tabular}{cccccc}
\hline $\begin{array}{c}\text { Deviation on detail } \\
\text { of ornamental }\end{array}$ & $\begin{array}{c}\text { more } \\
(-2)\end{array}$ & $\begin{array}{c}\text { a little more } \\
(-1)\end{array}$ & $\begin{array}{c}\text { perfect } \\
(0)\end{array}$ & $\begin{array}{c}\text { a little less } \\
(1)\end{array}$ & $\begin{array}{c}\text { less } \\
(2)\end{array}$ \\
\hline$\Delta \mathrm{do}_{1}=\mathrm{do}_{1}^{*}-\mathrm{do}_{1}$ & $-0.2 \times \mathrm{do}_{1}$ & $-0.1 \times \mathrm{do}_{1}$ & 0 & $0.1 \times \mathrm{do}_{1}$ & $0.2 \times \mathrm{do}_{1}$ \\
$\Delta \mathrm{do}_{2}=\mathrm{do}_{2}^{*}-\mathrm{do}_{2}$ & $+0.2 \times \mathrm{do}_{2}$ & $+0.1 \times \mathrm{do}_{2}$ & 0 & $-0.1 \times \mathrm{do}_{2}$ & $-0.2 \times \mathrm{do}_{2}$ \\
\hline
\end{tabular}

Table 5. The statistical results for the three cases of Validation 2.

\begin{tabular}{cccc}
\hline & Scenario 2.1 & Scenario 2.2 & Scenario 2.3 \\
\hline $\begin{array}{c}\text { Success Rate } \\
\text { Averaged numbers of apply the } \\
\text { recommendation module }\end{array}$ & $30 \%$ & $90 \%$ & $65 \%$ \\
\hline
\end{tabular}




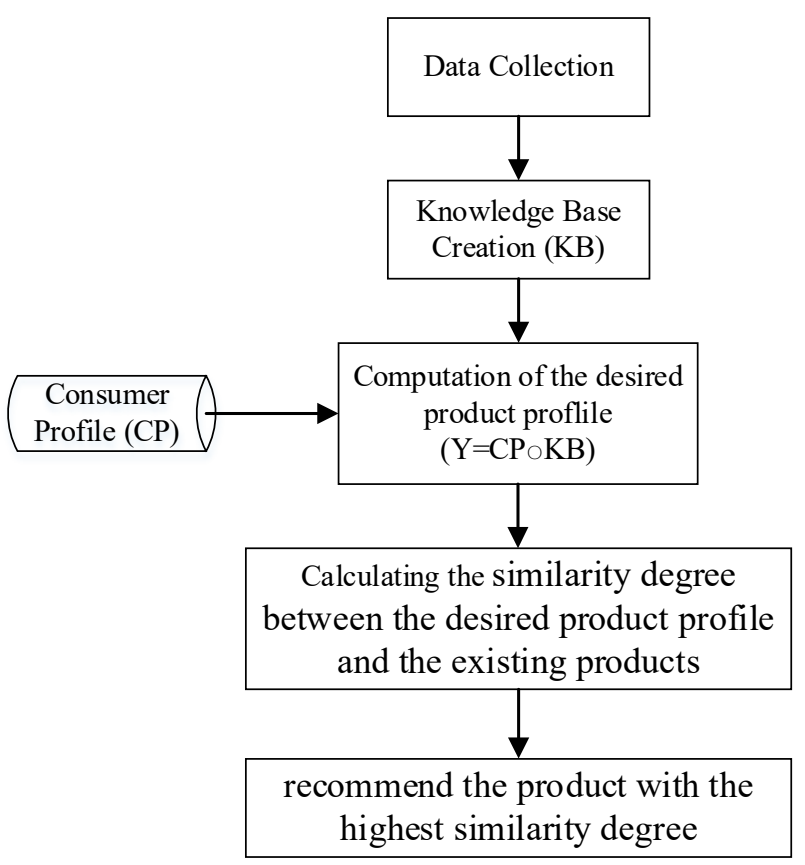

Fig. 1. General structure of the Knowledge-based Recommendation Module

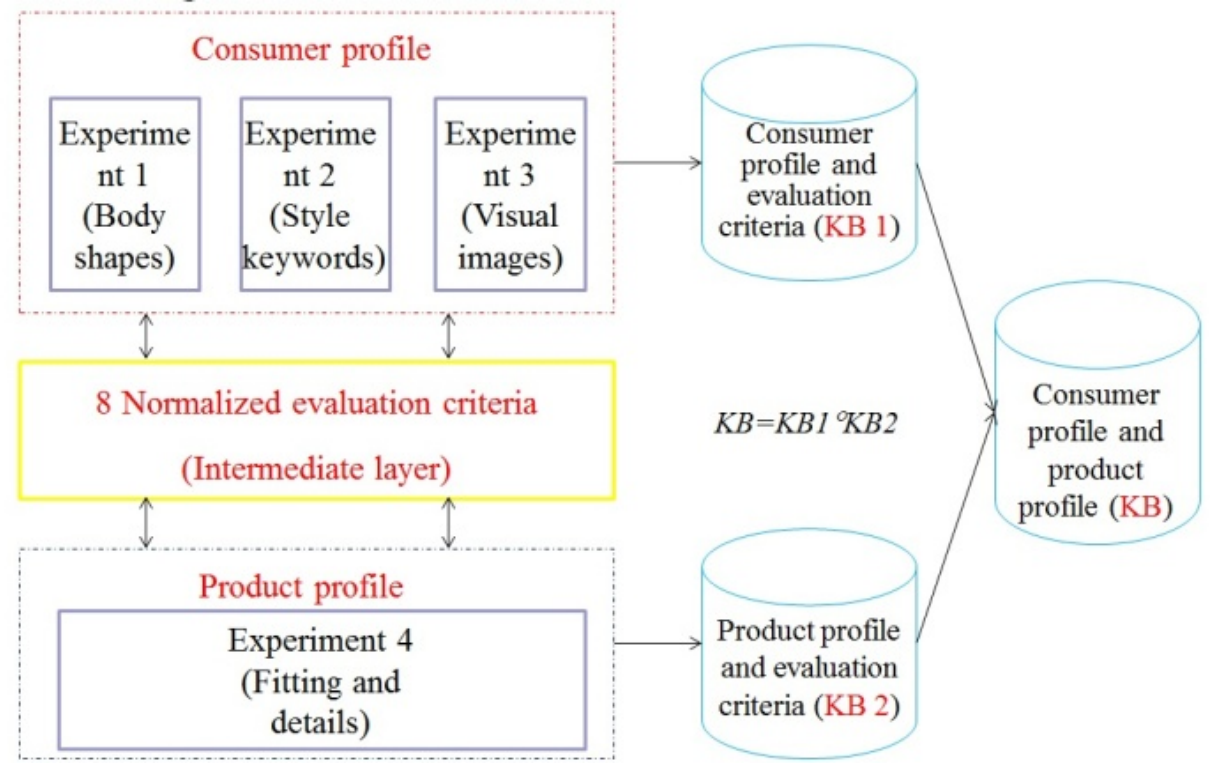

Fig. 2. The Relation of the Knowledge-based and Experiments 


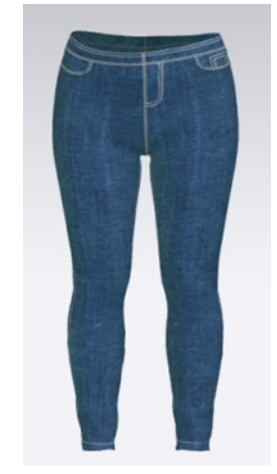

Fig. 3. Picture of $y 2$

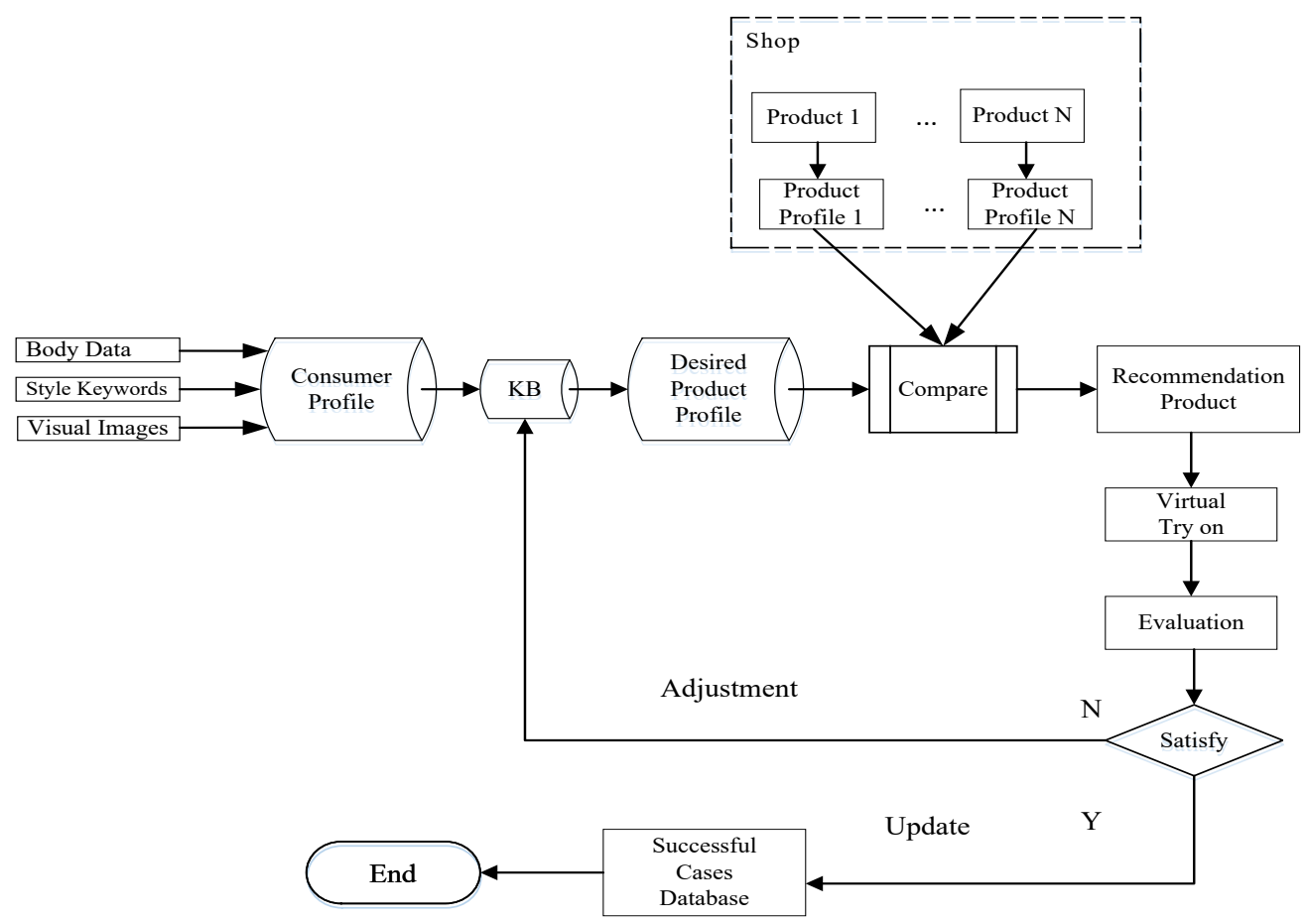

Fig. 4. General structure of the Knowledge Updating Module 


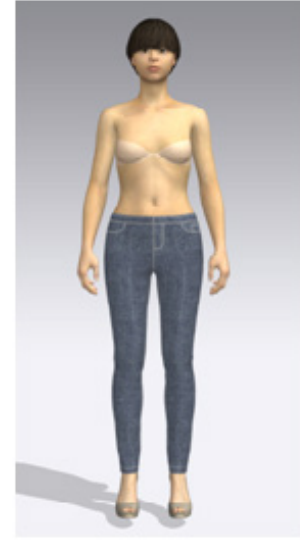

(a)|

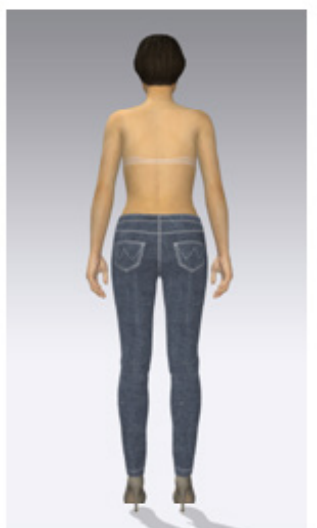

(b)

Fig. 5. virtual fitting effects of jeans by a specific female consumer

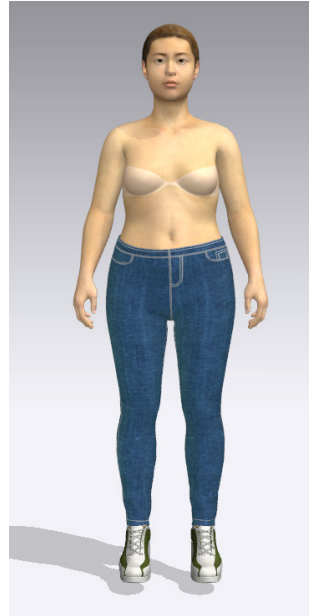

Fig. 6. virtual fitting effects of the product recommended by the Knowledge-based Recommendation Module

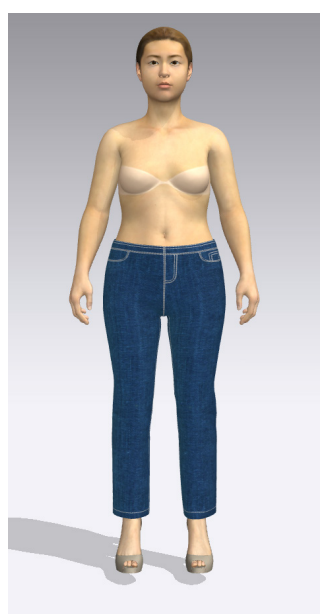

Fig. 7. virtual fitting effects of the product recommended by using the Knowledge Updating Module 